\title{
Impact of Energy and Access Methods on Extrahepatic Tumor Spreading and the Ablation Zone: An Ex vivo Experiment Using a Subcapsular Tumor Model
}

\author{
Jin Sil Kim, MD, Youngsun Ko, MD, Hyeyoung Kwon, MD, Minjeong Kim, MD, Jeong Kyong Lee, MD \\ All authors: Department of Radiology, College of Medicine, Ewha Womans University, Seoul, Korea
}

Objective: To evaluate the impact of energy and access methods on extrahepatic tumor spreading and the ablation zone in an ex vivo subcapsular tumor mimic model with a risk of extrahepatic tumor spreading.

Materials and Methods: Forty-two tumor-mimics were created in bovine liver blocks by injecting a mixture of iodine contrast material just below the liver capsule. Radiofrequency (RF) ablations were performed using an electrode placed parallel or perpendicular to hepatic surface through the tumor mimic with low- and high-power protocols (groups 1 and 2, respectively). Computed tomography (CT) scans were performed before and after ablation. The presence of contrast leak on the hepatic surface on CT, size of ablation zone, and timing of the first roll-off and popping sound were compared between the groups. Results: With parallel access, one contrast leak in group $1(1 / 10,10 \%)$ and nine in group $2(9 / 10,90 \%)(p<0.001)$ were identified on post-ablation CT. With perpendicular access, six contrast leaks were identified in each group $(6 / 11,54.5 \%)$. The first roll-off and popping sound were significantly delayed in group 1 irrespective of the access method $(p=0.002)$. No statistical difference in the size of the ablation zone of the liver specimen was observed between the two groups $(p=0.247)$. Conclusion: Low-power RF ablation with parallel access is proposed to be effective and safe from extrahepatic tumor spreading in RF ablation of a solid hepatic tumor in the subcapsular location. Perpendicular placement of an electrode to the capsule is associated with a risk of extrahepatic tumor spreading regardless of the power applied.

Keywords: Radiofrequency ablation; Thermal ablation; Experimental liver neoplasm; Neoplasm seeding

\section{INTRODUCTION}

Radiofrequency (RF) ablation is a localized thermal therapy used to eradicate tumors by heating the tissue. RF ablation plays an increasingly important role in the management of metastasis, including colorectal cancer as well as hepatocellular carcinoma (1). However, extrahepatic tumor seeding has been reported in $0.47-12 \%$ of cases of

Received August 21, 2018; accepted after revision November 8, 2018.

Corresponding author: Jeong Kyong Lee, MD, Department of Radiology, College of Medicine, Ewha Womans University, 1071 Anyangcheon-ro, Yangcheon-gu, Seoul 07985, Korea.

- Tel: (822) 2650-5174 • Fax: (822) 2650-5302

- E-mail: kyongmd@ewha.ac.kr

This is an Open Access article distributed under the terms of the Creative Commons Attribution Non-Commercial License (https://creativecommons.org/licenses/by-nc/4.0) which permits unrestricted non-commercial use, distribution, and reproduction in any medium, provided the original work is properly cited. thermal ablation (2-5). Several risk factors associated with extrahepatic tumor seeding after RF ablation have been identified, including subcapsular location, poor histological differentiation, tumor biopsy prior to RF ablation, larger tumor size, and direct puncture of the tumor $(2,3,6-8)$. Subcapsular location of the tumor as a risk factor for extrahepatic tumor seeding has been explained based on increased intratumoral pressure with subsequent rupture of the liver capsule and a higher risk of dissemination of viable tumor cells into the peritoneal cavity by direct puncture of the tumor without traversing the normal parenchyma $(2,9)$. Several studies have attempted to reduce tumor seeding after RF ablation of subcapsular tumors (1013). The reported methods to reduce tumor seeding and obtain adequate ablation margins were the no-touch wedge ablation technique and the angled-tip method $(10,11,14)$, which avoid direct puncture of the tumor. Low-power RF ablation has been reported as a power-applying protocol to 
avoid a rapid increase in intratumoral pressure and steam popping compared to that in high-power RF ablation (12, 13). Some studies have reported that the efficacy and safety of low-power RF ablation in tumor ablation was comparable to that of high-power RF ablation $(12,15)$. However, the role of low-power RF ablation in reducing tumor seeding after ablation of subcapsular tumors has not been clearly demonstrated, since tumor seeding is very rare and generally happens long after RF ablation.

Therefore, the aim of this study was to evaluate the impact of energy and access methods on extrahepatic tumor spreading and the ablation zone in an ex vivo subcapsular tumor mimic model with a risk of extrahepatic tumor spreading.

\section{MATERIALS AND METHODS}

\section{Preparation of the Tumor Mimic Model}

Four bovine livers were obtained from a slaughterhouse on the same day as RF ablation was performed for the experiments. The livers were cut into 42 blocks of $10 \times 10 \times$ $5 \mathrm{~cm}$ in size, excluding large vascular structures. Each block of liver tissue with the capsule on one side was packed in a rigid paper case with the bottom and one side left open for the injection of a mixture to create the tumor mimic.

For training in RF ablation, previous researchers developed tumor-mimic models in both ex vivo and in vivo settings $(16,17)$. A mixture containing $3 \%$ agarose, $3 \%$ cellulose, $7 \%$ glycerol, and $0.05 \%$ methylene blue was used with reference to a previous study $(16,17)$. The mixture was prepared by mixing $6 \mathrm{~g}$ of agarose, $6 \mathrm{~g}$ of cellulose, 14 $\mathrm{mL}$ of glycerol, and $100 \mathrm{mg}$ of methylene blue for a total volume of $200 \mathrm{~mL}$ with distilled water. To solubilize the mixture, the solution was heated to $95^{\circ} \mathrm{C}$ for approximately 5 minutes using a heating mantle. Aliquots of $200 \mathrm{~mL}$ were mixed with $180 \mathrm{~mL}$ of contrast media (iopamidol, Pamiray 300, Dongkuk Pharmaceutical, Seoul, Korea). The mixture was sealed in airtight tubes and allowed to cool to $22 \pm 2^{\circ} \mathrm{C}$. After polymerization of the mixture into a solid, gelatinlike material, the tubes were stored at $22 \pm 2^{\circ} \mathrm{C}$. Before injection, $200 \mathrm{~mL}$ of the aliquots was reheated to $65^{\circ} \mathrm{C}$ in a heating mantle.

A hyperechoic and spherical tumor mimic measuring approximately $1.5 \mathrm{~cm}^{2}$ in area was created by injecting 2 $\mathrm{mL}$ of the mixture into the subcapsular portion of a liver block through an 18 gauge intravenous angiocatheter by placing the catheter tip $5 \mathrm{~mm}$ from the liver capsule under

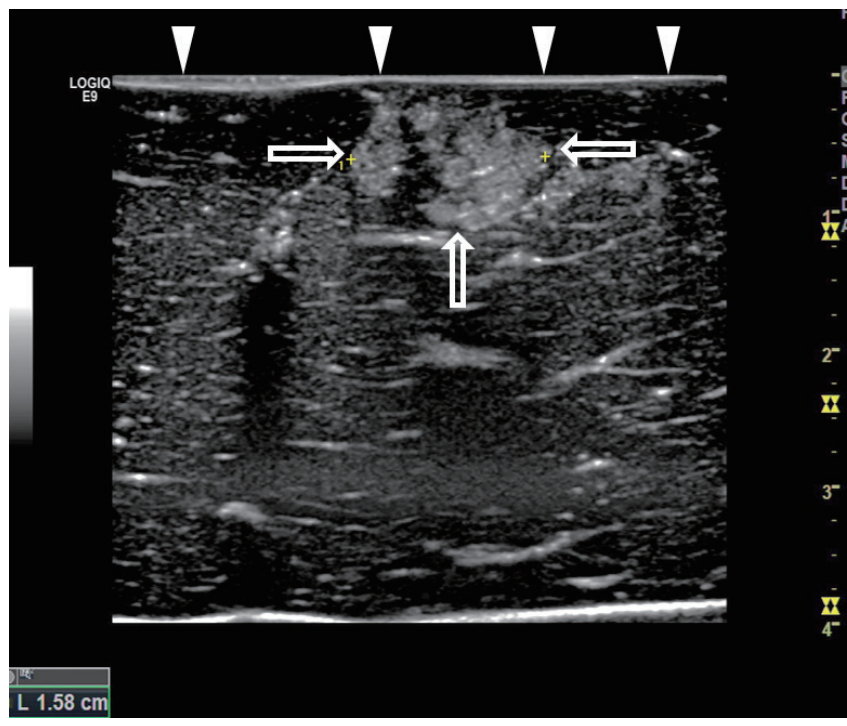

Fig. 1. US of subcapsular tumor mimic model. US image reveals hyperechoic mass-like lesion (arrows) measuring approximately $1.6 \mathrm{~cm}$ in diameter just below liver capsule (arrowheads). US = ultrasound

ultrasound (US) guidance (Fig. 1). The diameter of each tumor mimic measured on US was recorded.

\section{CT Examination}

Computed tomography (CT) scans were obtained for all tumor mimic models before and after RF ablation using a 128-slice multi-detector-row CT scanner (Somatom definition flash, Siemens Healthcare, Forchheim, Germany) with imaging parameters as follows: $128 \times 0.6 \mathrm{~mm}$ collimation, $1 \mathrm{~mm}$ slice thickness, $120 \mathrm{kVp}$, and $120 \mathrm{~mA}$.

\section{RF Ablation}

Liver blocks with a tumor mimic were placed on a noncurrent plate with a grounding pad fixed to the side of the plate filled with saline. A 200-watt RF generator with an impedance-controlled pulsed current (VIVA RF system; STARmed, Goyang, Korea) was used for all ablations. The starting impedance was controlled by maintaining the distance between the grounding pad and the liver blocks and set at 80-83 $\Omega$ (Fig. 2). A 15-gauge monopolar, internally-cooled electrode (Proteus; STARmed) with a 3.0 $\mathrm{cm}$ exposed tip was placed in the tumor mimic by freehand technique under US guidance using a $7 \mathrm{MHz}$ linear probe.

\section{RF Ablation Protocols}

RF ablation was performed for 5 minutes following two different protocols with either the parallel or perpendicular access method. In experiment A with parallel access, the electrode was inserted parallel to the liver capsule 


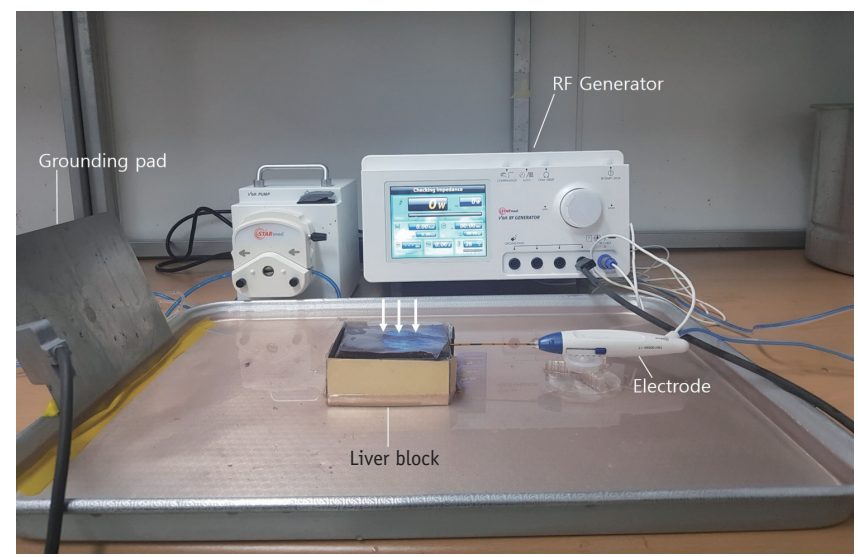

A

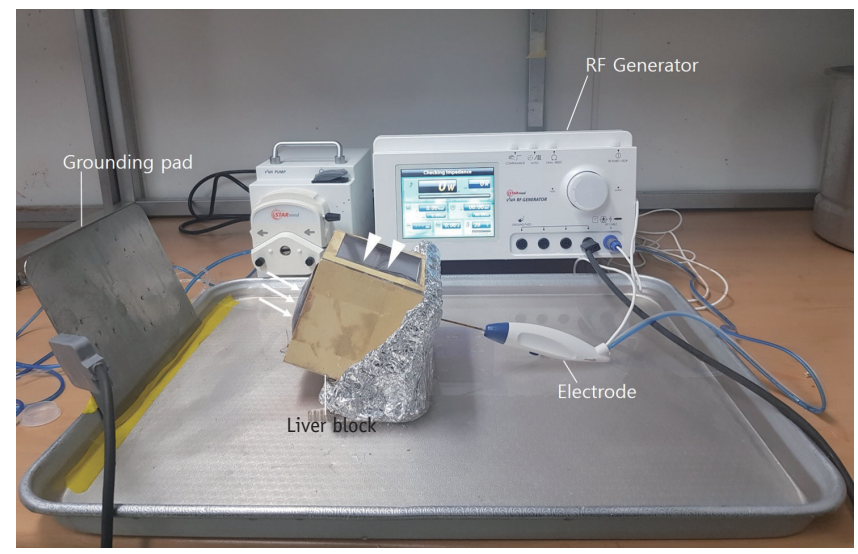

B

Fig. 2. Photographs of experimental models and RF ablation system.

Sectioned bovine liver block with tumor mimic created in subcapsular portion is placed in water plate. (A) Electrode is inserted at lateral surface of liver block and advanced parallel to capsule (arrows). (B) Electrode is inserted at bottom of liver block opposite capsule and advanced through tumor mimic towards capsule (arrows). Lateral side of paper case is open for US guidance (arrowheads). RF = radiofrequency

at $5 \mathrm{~mm}$ distance from the capsule through the center of the tumor mimic (Figs. 2A, 3A). In experiment $B$ with perpendicular access, the electrode was inserted towards the capsule through the center of the tumor mimic by placing the electrode tip at a distance of $5 \mathrm{~mm}$ from the liver capsule (Figs. 2B, 3B). In group 1 with the low-power protocol, the power of the generator was initially set at 40 watts for 1 minute and then increased to 70 watts in increments of 10 watts every 1 minute. For the remaining 1 minute in the low-power protocol, 70 watts power was maintained under the impedance-control mode (12). In group 2 with the high-power protocol, the power of the generator increased sharply to reach the maximum wattage under the impedance-control mode (18). The low-power

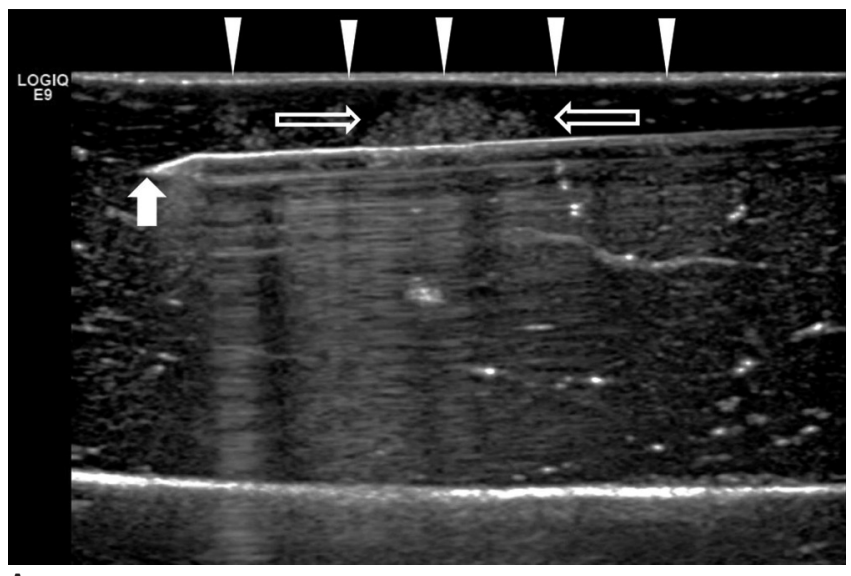

A

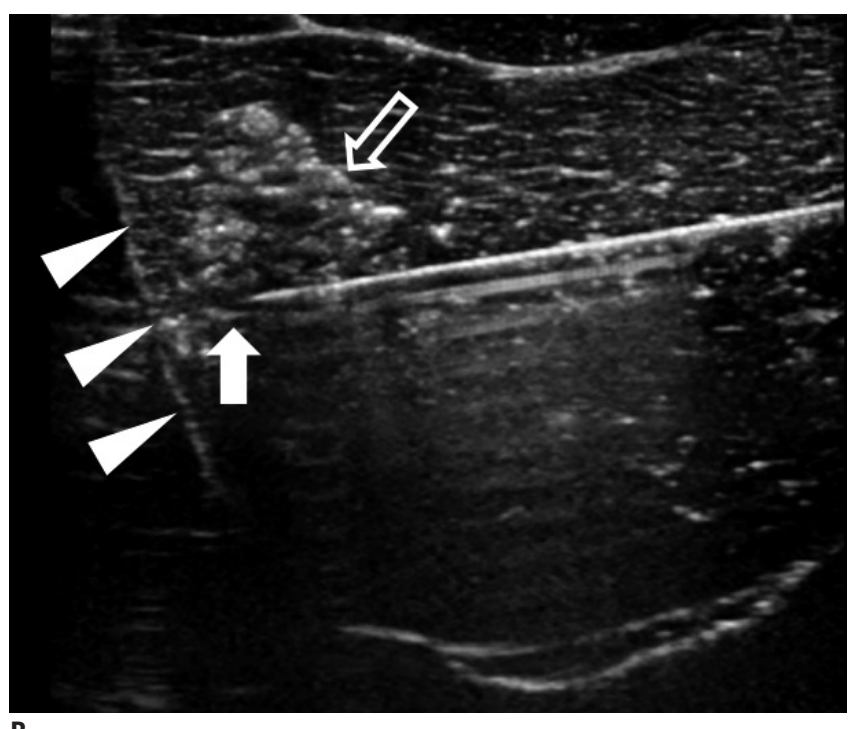

B

Fig. 3. US of parallel and perpendicular access methods.

A. US image illustrating parallel access method. Electrode (arrow) is placed parallel to liver capsule (arrowheads) through tumor mimic (open arrows). B. US image illustrating perpendicular access method. Tip of electrode (arrow) extends towards liver capsule (arrowheads) through tumor mimic (open arrow).

protocol with parallel access was applied in 10 tumor mimic models, low-power protocol with perpendicular access in 11 models, high-power protocol with parallel access in 10 models, and high-power protocol with perpendicular access in 11 models. The temperature, power, and impedance were continuously checked. The time of the first roll-off and popping sound was recorded.

\section{Evaluation of the CT Images and Liver Specimens}

The CT images were reviewed by two radiologists without any information about the ablation protocol in consensus for the presence of a contrast leak from the tumor mimics towards the upper surface of the liver blocks. A contrast 
leak was confirmed when high-attenuation contrast was observed on the capsule on the upper surface of a liver block on the post-ablation images in comparison to the pre-ablation images at the same levels.

The liver specimens were sectioned parallel to the long axis of the electrode insertion tract. The ablation zone size was measured based on two diameters (i.e., the longest diameter and one perpendicular diameter) of the specimens. For perpendicular access, the transverse diameter of the ablation zone just beneath the liver capsule was also measured.

\section{Statistical Analysis}

All statistical analyses were performed using commercially available software (SPSS version 21.0; IBM Corp., Armonk, NY, USA). The results are expressed as means \pm standard deviation. The following were compared between group 1 and group 2 in each experiment ( $A$ and $B$ ): the presence of a contrast leak, size of the ablation zone, and timing of the first roll-off and popping. Categorical values were compared by the chi-square test and numerical values were analyzed by the Mann-Whitney U-test. A $p$ value of $<0.05$ was defined as significant.

\section{RESULTS}

The size of the tumor mimics as measured by US was not significantly different between the groups $(1.6 \pm 0.5$ $\mathrm{cm}^{2}$ in group 1 , and $1.5 \pm 0.3 \mathrm{~cm}^{2}$ in group 2, $p=0.971$ ). The results of experiment $A$ are summarized in Table 1. In experiment A with parallel access, one contrast leak (1/10, $10 \%$ ) was identified on CT in group 1 and nine contrast leaks $(9 / 10,90 \%)$ were identified in group $2(p<0.001)$ (Figs. 4, 5). The first roll-off and popping sound occurred with delayed timing in group 1 compared to that in group 2 (229.0 seconds vs. $89.4 \pm 25.8$ seconds, the first roll-off in group 1 and group 2, respectively; $135.3 \pm 40.6$ seconds vs. $38.0 \pm 16.4$ seconds, $p=0.002$, popping sound in group 1 and group 2, respectively). The first roll-off occurred after the initial popping sound during all ablations. In group 1 , six tumor mimic models did not have popping sounds within 5 minutes and one of the six models without a popping sound had a contrast leak. In group 1, only one tumor mimic model had roll-off within 5 minutes. The area of the ablation zones did not significantly differ between group 1 and group $2\left(9.7 \pm 1.5 \mathrm{~cm}^{2}\right.$ in group 1 and $8.7 \pm 1.4 \mathrm{~cm}^{2}$ in group $2, p=0.247)$.

The results of experiment B are summarized in Table 2 .

Table 1. Comparison of RF Ablation between Two Groups with Parallel Access

\begin{tabular}{lccc}
\hline \multicolumn{1}{c}{ Measurement } & Group 1 $(\mathrm{n}=10)$ & Group 2 $(\mathrm{n}=10)$ & $P$ \\
\hline Pre-ablation size of tumor mimic model $\left(\mathrm{cm}^{2}\right)$ & $1.6 \pm 0.5$ & $1.5 \pm 0.3$ & 0.971 \\
Initial tissue impedance $(\Omega)$ & $82.2 \pm 1.1$ & $82.2 \pm 1.61$ & 0.971 \\
No. of contrast leak $(\mathrm{n})^{*}(\%)$ & $1(10)$ & $9(90)$ & $<0.001$ \\
Time of first popping sound $(\mathrm{sec})$ & $135.3 \pm 40.6^{\dagger}$ & $38.0 \pm 16.4$ & 0.002 \\
First roll-off time $(\mathrm{sec})$ & $229.0^{\ddagger}$ & $89.4 \pm 25.8$ & $\mathrm{NA}$ \\
Ablation zone $\left(\mathrm{cm}^{2}\right)$ & $9.7 \pm 1.5$ & $8.7 \pm 1.4$ & 0.247 \\
\hline
\end{tabular}

Unless otherwise indicated, data are mean \pm standard deviation. Group 1 means RF ablation with low RF protocol, Group 2 means RF ablation with high RF protocol. $p$ value could not be obtained since Group 1 had only one case with roll-off. *Values are number of tumor mimic models, with percentage in parentheses, ${ }^{\dagger} n=4,{ }^{\ddagger} n=1$. NA $=$ not applicable, $R F=$ radiofrequency

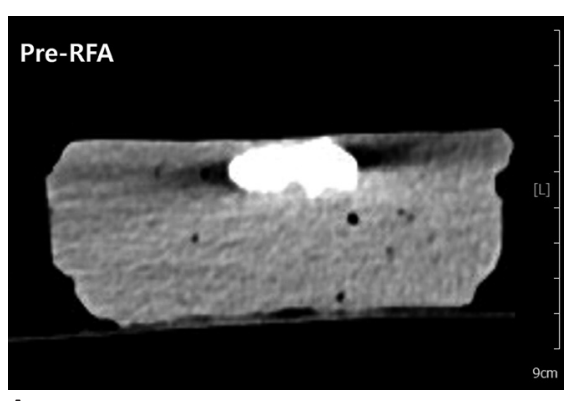

A
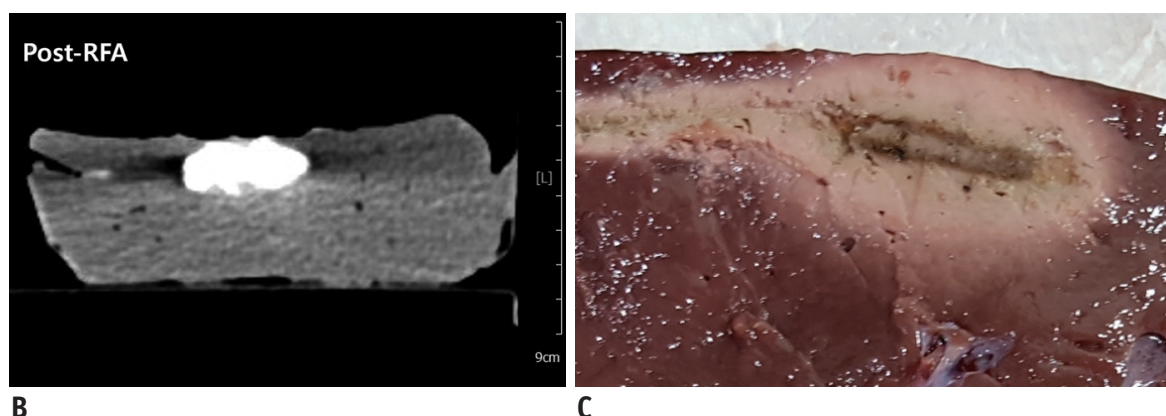

C

Fig. 4. RF ablation with low-power protocol and parallel access.

A, B. Pre- and post-RF ablation CT images. Post-RF ablation CT image (B) reveals no visible contrast leak on upper hepatic surface, compared to that observed on pre-RF ablation CT image (A). C. Liver specimen corresponding to CT images. Analysis of specimen revealed no visible contrast leak on surface of liver. $\mathrm{CT}$ = computed tomography, $\mathrm{RF}=$ radiofrequency 


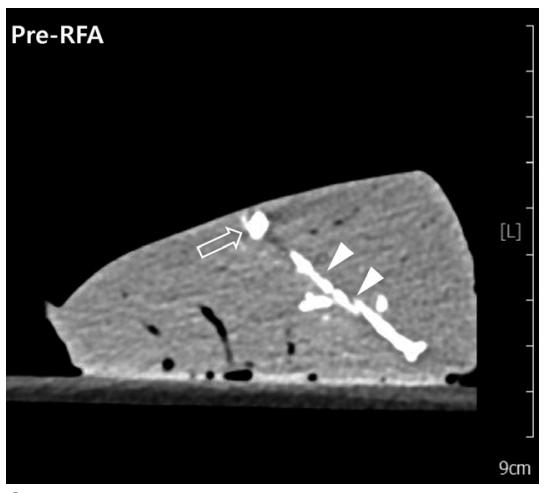

A

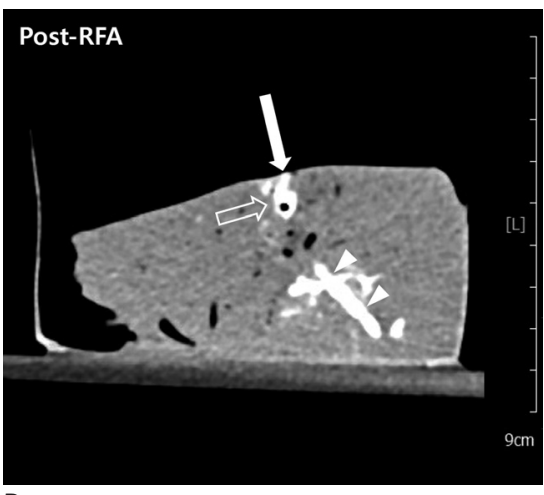

B

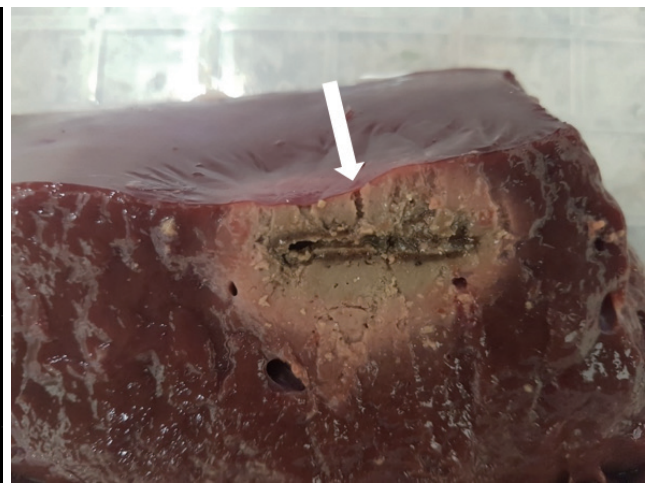

C

Fig. 5. RF ablation with high-power protocol and parallel access.

A, B. Pre- and post-RF ablation CT images. Post-RF ablation CT image (B) reveals contrast leak on hepatic surface (arrow) compared to that observed on pre-RF ablation CT image (A). High-density lines (arrowheads) inferior to tumor mimic (open arrows) is track through which tumor mimic material was injected. C. Liver specimen corresponding to CT images. Specimen image illustrates track of contrast leak corresponding to tract on post-RF ablation CT (arrow).

Table 2. Comparison of RF Ablation between Two Groups with Perpendicular Access

\begin{tabular}{|c|c|c|c|}
\hline Measurement & Group $1(n=11)$ & Group $2(n=11)$ & $P$ \\
\hline Pre-ablation size of tumor mimic model $\left(\mathrm{cm}^{2}\right)$ & $1.6 \pm 0.5$ & $1.6 \pm 0.4$ & 0.699 \\
\hline Initial tissue impedance $(\Omega)$ & $81.1 \pm 2.3$ & $82.9 \pm 2.1$ & 0.076 \\
\hline No. of contrast leak* $(\%)$ & $6(54.5)$ & $6(54.5)$ & 1.000 \\
\hline Time of first popping sound (sec) & $174.2 \pm 51.5$ & $20.1 \pm 8.8$ & $<0.001$ \\
\hline First roll-off time (sec) & $231.4 \pm 47.9^{\dagger}$ & $78.5 \pm 29.6$ & $<0.001$ \\
\hline Ablation zone $\left(\mathrm{cm}^{2}\right)$ & $10.4 \pm 1.2$ & $9.9 \pm 2.4$ & 0.401 \\
\hline Transverse diameter of ablation zone below capsule $(\mathrm{cm})$ & $2.2 \pm 0.9$ & $1.5 \pm 0.4$ & 0.065 \\
\hline
\end{tabular}

Unless otherwise indicated, data are mean and standard deviation. Group 1 means RF ablation with low RF protocol, Group 2 means RF ablation with high RF protocol. *Values are number of tumor mimic models, with percentage in parentheses, ${ }^{\dagger} n=7$

In experiment $B$ with perpendicular access, six contrast leaks $(6 / 11,54.5 \%)$ were identified on CT in group 1 and six contrast leaks $(6 / 11,54.5 \%)$ were identified in group 2 $(p=1.00)$. Similar to that in experiment A, the first roll-off and popping sound occurred with delayed timing in group 1 compared to that in group $2(231.4 \pm 47.9$ seconds vs. 78.5 \pm 29.6 seconds, $p<0.001$, the first roll-off in group 1 and group 2, respectively; $174.2 \pm 51.5$ seconds vs. $20.1 \pm 8.8$ seconds, $p<0.001$, popping sound in group 1 and group 2 , respectively). In group 1 , four tumor mimic models did not have roll-off within 5 minutes. The area of the ablation zones did not significantly differ between groups (10.4 \pm 1.2 $\mathrm{cm}^{2}$ vs. $\left.9.9 \pm 2.4 \mathrm{~cm}^{2}, p=0.401\right)$. The transverse diameter of the ablation zone measured just beneath the capsule was larger in group 1 than in group 2 and exhibited borderline statistical significance $(2.2 \pm 0.9 \mathrm{~cm}$ in group 1 and $1.5 \pm$ $0.4 \mathrm{~cm}$ in group 2, $p=0.065$ ) (Fig. 6).

A comparative analysis of experiments $A$ and $B$ revealed a tendency for the number of contrast leaks to increase in group 1 and a decrease in group 2. However, regardless of the access method, contrast leaks were still more frequent in group 2 than in group $1(7 / 21,33 \%$ in group 1 and 15/21, $71 \%$ in group $2, p=0.029$ ). The first roll-off and popping sound were delayed in group 1 compared to that in group 2 in both access methods. Nevertheless, contrast leak was similarly present in both groups with perpendicular access irrespective of the occurrence of the delayed popping sound.

\section{DISCUSSION}

Extrahepatic tumor spreading is a fatal complication, which leads to reluctance in treating subcapsular tumors with RF ablation. Considering the increase in intratumoral pressure with subsequent rupture of the hepatic capsule as a cause of extrahepatic tumor spreading, the low-power $\mathrm{RF}$ ablation protocol with avoidance of steep elevation of intratumoral pressure may be a good strategy for treating subcapsular tumors. A previous study suggested that initiation of a modified RF ablation protocol at 40 watts with an incremental increase of 10 watts every 1 minute could reduce rapid and scattered recurrence in the liver after RF ablation of hepatocellular carcinoma compared to 

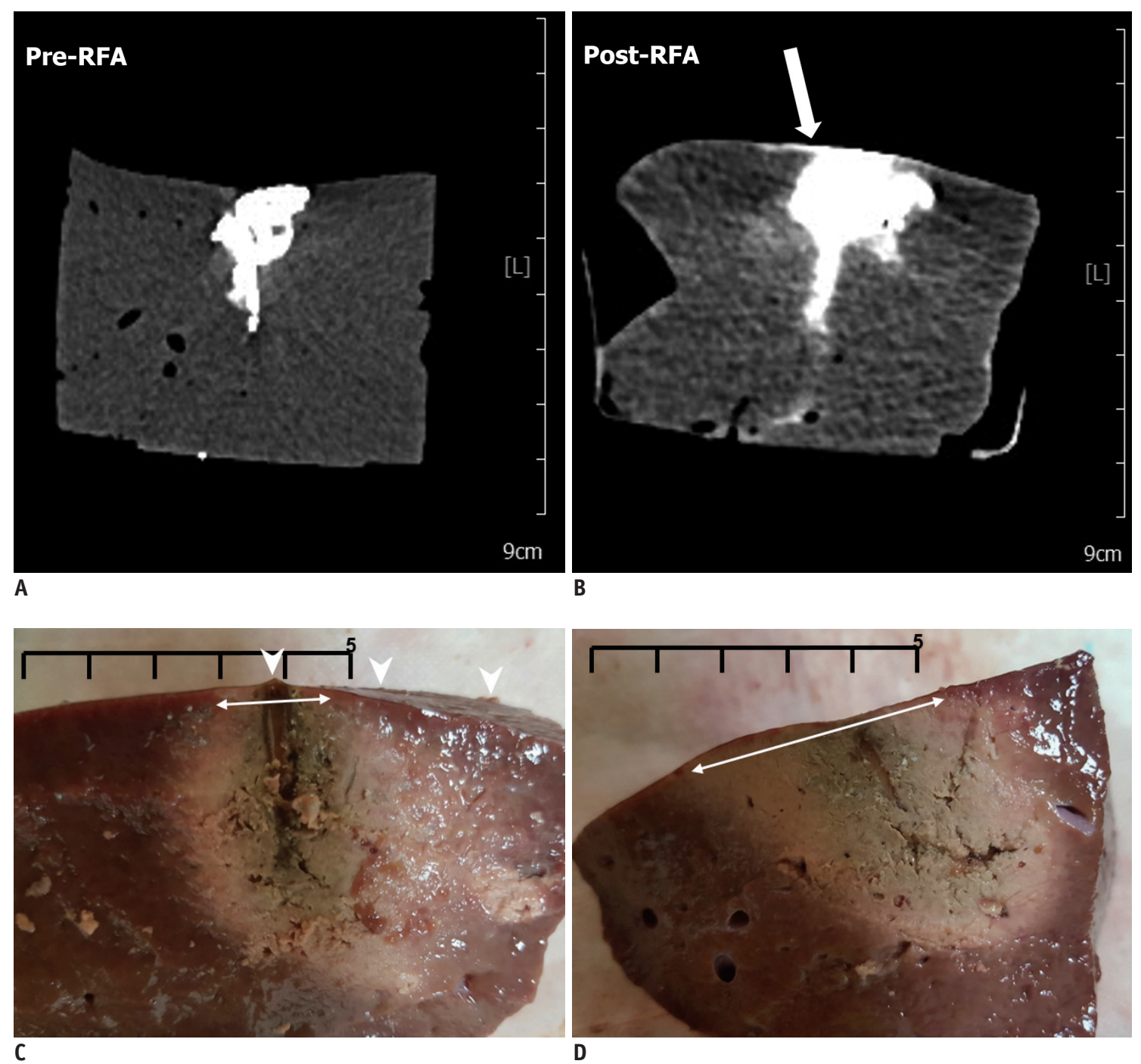

Fig. 6. RF ablation with high-power protocol and perpendicular access.

A, B. Pre- and post-RF ablation CT images. Post-RF ablation CT image (B) reveals contrast leak on hepatic surface after RF ablation, compared to that observed on pre-RF ablation CT image (A) (arrow). C. Liver specimen corresponding to CT images. Analysis of specimen reveals contrast leak on hepatic surface (arrowheads). D. Another liver specimen with low-power protocol and perpendicular access. Analysis of specimen reveals no contrast leak on hepatic surface, and diameter of ablation zone just beneath capsule is larger than that of specimen shown in (C) (double-headed arrows on C, D) (Scale bar: $5 \mathrm{~cm}$ ).

that associated with the conventional high-power protocol $(13,19)$. However, the role of the low-power protocol in the reduction of extrahepatic tumor spreading is not known for RF ablation of hepatic subcapsular tumors. It is not easy to prove the advantage of the low-power protocol in a clinical setting, as the incidence of extrahepatic tumor seeding after RF ablation of subcapsular tumors is low and usually happens long after RF ablation. Previous studies reporting a low risk of tumor seeding after RF ablation of subcapsular tumors included superficial subcapsular tumors, which can be ablated with intervening parenchyma to avoid direct puncture of the tumor $(10,20)$. A subcapsular tumor in the anterior segment of the liver can generally be approached with parallel access to avoid direct puncture of the tumor. However, an electrode cannot be easily placed with parallel access in the posterior segment of the liver, particularly segment VII. Insertion of the electrode facing the capsule in perpendicular access cannot be avoided. Nevertheless, extrahepatic tumor spreading from RF ablation of subcapsular tumors located in the posterior margin of the hepatic posterior segment is not well known.

We created a novel experimental model capable of demonstrating extrahepatic tumor spreading on CT imaging immediately after RF ablation. Our model may provide clinically significant solutions to the safety issues regarding RF ablation of subcapsular tumors. 
The subcapsular tumor mimic model can demonstrate extrahepatic tumor spreading according to the power application or electrode access methods. In this study, the low-power protocol with parallel access resulted in fewer contrast leaks than did perpendicular access or the highpower protocol. Interestingly, an increasing tendency of contrast leaks was observed in the low-power protocol with perpendicular access, which simulated the route of electrode placement for a subcapsular tumor located in the posterior margin of the hepatic posterior segment, compared to that in the low-power protocol with parallel access. The possible explanation behind this observation is that RF energy concentrates first on the tip of an electrode and the capsule facing the tip can be exposed to relatively high temperature and pressure instantaneously, such that the capsule can be easily disrupted even with the lowpower protocol and a distance of $5 \mathrm{~mm}$ between the tip and the capsule. The tumor mimics can change to a liquid state rapidly using a high initial temperature with the highpower protocol compared to with the low-power protocol, which may increase the rate of contrast leakage with the high-power protocol. However, our study revealed that the rate of contrast leakage with the low-power protocol was equal to that with the high-power protocol in perpendicular access, which would suggest that the injury of the capsule might have played a more important role for contrast leakage than did the fluid content of the tumor mimics. In the case of perpendicular access, the diameter of the ablation zone just beneath the capsule was smaller in the high-power protocol than in the low-power protocol, as the temperature increased rapidly around the tip and heat did not spread far in the high-power protocol due to the creation of charred tissue and frequent roll-off. This phenomenon may lead to an insufficient ablative margin adjacent to the capsule and a higher rate of local tumor progression. Therefore, RF ablation of the subcapsular tumor that is not feasible by parallel access should be performed using the low-power protocol without penetrating the tumor using multiple electrodes following the "no touch technique" to prevent spreading of the extrahepatic tumor and creating an insufficient ablative margin.

The mean ablation area increased slightly when RF ablation was performed following the low-power protocol compared to that when following the high-power protocol, although the increase was not statistically significant. High-power protocols are widely used because a large ablation area can be achieved with a short ablation time
(21). However, in impedance-controlled RF ablation, the high-power protocol could induce increases in impedance resulting in repeated impedance spiking and an automatic cool-down cycle (i.e., roll-off), which could decrease the treatment efficiency $(12,22)$. Consequently, it is proposed that low-power RF ablation could provide comparable therapeutic effects to those achieved by high-power RF ablation.

The association of the popping phenomenon with tumor dispersion remains controversial (23). Some reports have suggested that steam popping was a sign of an abrupt increase in the intratissue pressure, which could lead to injury of the liver capsule or hepatic vessels $(12,19,24)$. As in the previous study, steam popping occurred at a relatively delayed rate with the low-power protocol compared to that with the high-power protocol (12). However, our study demonstrated the occurrence of contrast leak in the highand low-power protocols with perpendicular access to a similar extent regardless of the delayed popping sound. Consequently, it is hypothesized that the popping sound may not be related to extrahepatic tumor spreading, such as the rupture of the liver capsule. A prospective study also reported that the popping sound during RF ablation was not associated with an increased risk of early tumor progression or poorer patient survival (23).

There are several limitations to this study. First, as the present study involved an ex vivo setting, it could not reflect in vivo physiology such as the heat-sink effect. In vivo experiments are necessitated to confirm the ex vivo observations of this study. Second, gelatin-like tumor mimics change to a liquid state at the high temperature of RF ablation and the liquid leaks more easily than the solid state to exaggerate the rate of extrahepatic leakage. Therefore, this study observed a higher rate of contrast leak than the reported incidence of extrahepatic seeding of solid tumors. Third, there was no consideration of other risk factors of extrahepatic tumor spreading, such as the histological degree of the tumor.

In summary, the low-power RF ablation protocol with an electrode placed parallel to the liver capsule resulted in a significantly lower rate of contrast leak into the hepatic outer surface during the treatment of a subcapsular tumor than did the high-power protocol. RF ablation with an electrode placed perpendicular to liver capsule resulted in a high rate of contrast leak in both the high- and low-power protocols. The size of the ablation zone with the low-power protocol was similar to that of the high-power protocol. 
In conclusion, the low-power protocol with parallel access is proposed to be effective and safe from extrahepatic tumor spreading in RF ablation of solid subcapsular hepatic tumors. Perpendicular placement of an electrode to the capsule carries a risk of extrahepatic tumor spreading regardless of the type of energy-supplying protocol. Therefore, to prevent extrahepatic tumor spreading and secure an adequate ablative margin around the capsule, the low-power protocol with parallel access is strongly advised.

\section{Conflicts of Interest}

The authors have no potential conflicts of interest to disclose.

\section{ORCID iDs \\ Jeong Kyong Lee \\ https://orcid.org/0000-0002-5507-0140 \\ Jin Sil Kim \\ https://orcid.org/0000-0002-3321-2507}

\section{REFERENCES}

1. Ahmed M, Brace CL, Lee Jr FT, Goldberg SN. Principles of and advances in percutaneous ablation. Radiology 2011;258:351369

2. Llovet JM, Vilana R, Brú C, Bianchi L, Salmeron JM, Boix L, et al. Increased risk of tumor seeding after percutaneous radiofrequency ablation for single hepatocellular carcinoma. Hepatology 2001;33:1124-1129

3. Shirai K, Tamai H, Shingaki N, Mori Y, Moribata K, Enomoto S, et al. Clinical features and risk factors of extrahepatic seeding after percutaneous radiofrequency ablation for hepatocellular carcinoma. Hepatol Res 2011;41:738-745

4. Yu J, Liang P, Yu XL, Cheng ZG, Han ZY, Dong BW. Needle track seeding after percutaneous microwave ablation of malignant liver tumors under ultrasound guidance: analysis of 14-year experience with 1462 patients at a single center. Eur J Radiol 2012;81:2495-2499

5. Kim JW, Shin SS, Heo SH, Hong JH, Lim HS, Seon HJ, et al. Ultrasound-guided percutaneous radiofrequency ablation of liver tumors: how we do it safely and completely. Korean J Radiol 2015;16:1226-1239

6. Livraghi T, Solbiati L, Meloni MF, Gazelle GS, Halpern EF, Goldberg SN. Treatment of focal liver tumors with percutaneous radio-frequency ablation: complications encountered in a multicenter study. Radiology 2003;226:441-451

7. Teratani T, Yoshida H, Shiina S, Obi S, Sato S, Tateishi R, et al. Radiofrequency ablation for hepatocellular carcinoma in so-called high-risk locations. Hepatology 2006;43:1101-1108

8. Zhong-yi Z, Wei Y, Kun Y, Ying D, Wei W, Jung-Chieh L, et al. Needle track seeding after percutaneous radiofrequency ablation of hepatocellular carcinoma: 14-year experience at a single centre. Int J Hyperthermia 2017;33:454-458

9. Baldan A, Marino D, DE Giorgio M, Angonese C, Cillo U, D'Alessandro $A$, et al. Percutaneous radiofrequency thermal ablation for hepatocellular carcinoma. Aliment Pharmacol Ther 2006;24:1495-1501

10. Patel PA, Ingram L, Wilson ID, Breen DJ. No-touch wedge ablation technique of microwave ablation for the treatment of subcapsular tumors in the liver. $J$ Vasc Interv Radiol 2013;24:1257-1262

11. Park SI, Kim IJ, Lee SJ, Shin MW, Shin WS, Chung YE, et al. Angled cool-tip electrode for radiofrequency ablation of small superficial subcapsular tumors in the liver: a feasibility study. Korean J Radiol 2016;17:742-749

12. Choe J, Kim KW, Kim YI, Chung JW, Huh J, Park J, et al. Feasibility of a low-power radiofrequency ablation protocol to delay steam popping. J Vasc Interv Radiol 2016;27:268-274

13. Kotoh K, Enjoji M, Arimura E, Morizono S, Kohjima M, Sakai $\mathrm{H}$, et al. Scattered and rapid intrahepatic recurrences after radio frequency ablation for hepatocellular carcinoma. World J Gastroenterol 2005;11:6828

14. Lee DH, Lee JM. Recent advances in the image-Guided tumor ablation of liver malignancies: radiofrequency ablation with multiple electrodes, real-time multimodality fusion imaging, and new energy sources. Korean J Radiol 2018;19:545-559

15. Macatula TC, Lin CC, Lin CJ, Chen WT, Lin SM. Radiofrequency ablation for hepatocellular carcinoma: use of low vs maximal radiofrequency power. Br J Radiol 2012;85:e102-109

16. Scott DJ, Young WN, Watumull LM, Lindberg G, Fleming JB, Rege RV, et al. Development of an in vivo tumor-mimic model for learning radiofrequency ablation. J Gastrointest Surg 2000;4:620-625

17. Hildebrand P, Kleemann M, Roblick U, Mirow L, Bruch HP, Bürk C. Development of a perfused ex vivo tumor-mimic model for the training of laparoscopic radiofrequency ablation. Surg Endosc 2007;21:1745-1749

18. Kim JH, Kim PN, Won HJ, Shin YM. Percutaneous radiofrequency ablation using internally cooled wet electrodes for the treatment of hepatocellular carcinoma. AJR Am J Roentgenol 2012;198:471-476

19. Kotoh K, Nakamuta M, Morizono S, Kohjima M, Arimura E, Fukushima $M$, et al. A multi-step, incremental expansion method for radio frequency ablation: optimization of the procedure to prevent increases in intra-tumor pressure and to reduce the ablation time. Liver Int 2005;25:542-547

20. Kim YJ, Raman SS, Yu NC, Busuttil RW, Tong M, Lu DS. Radiofrequency ablation of hepatocellular carcinoma: can subcapsular tumors be safely ablated? AJR Am J Roentgenol 2008;190:1029-1034

21. Pereira PL, Trübenbach J, Schenk M, Subke Jr, Kroeber S, Schaefer I, et al. Radiofrequency ablation: in vivo comparison of four commercially available devices in pig livers. Radiology 2004;232:482-490

22. Cua IHY, Lin CC, Lin CJ, Chen WT, Hsu CW, Chen YC, et al. 
Treatment of hepatocellular carcinoma using internally cooled electrodes. A prospective comparison of modified automated vs. manual pulsed radiofrequency algorithms. Oncology 2007;72:76-82

23. Fernandes ML, Lin CC, Lin CJ, Chen WT, Lin SM. Prospective study of a 'popping' sound during percutaneous radiofrequency ablation for hepatocellular carcinoma. J Vasc Interv Radiol 2010;21:237-244

24. Iida H, Aihara T, Ikuta S, Yamanaka N. Effectiveness of impedance monitoring during radiofrequency ablation for predicting popping. World J Gastroenterol 2012;18:5870-5878 